2017-04-21

Mechanism of adsorption of actives onto microporous functionalised calcium carbonate (FCC)

Levy, CL

http://hdl.handle.net/10026.1/9157

10.1007/s10450-017-9880-7

Adsorption

Springer Science and Business Media LLC

All content in PEARL is protected by copyright law. Author manuscripts are made available in accordance with publisher policies. Please cite only the published version using the details provided on the item record or document. In the absence of an open licence (e.g. Creative Commons), permissions for further reuse of content should be sought from the publisher or author. 
"This is the author's accepted manuscript. The final published version of this work is published by Springer in Adsorption, 2017 available at: http://dx.doi.org/10.1007/s10450-017-9880-7 . This work is made available in accordance with the publisher's policies. Please refer to any applicable terms of use of the publisher.”

\title{
Mechanism of adsorption of actives onto microporous functionalised calcium carbonate (FCC)
}

\author{
Charlotte L. Levy • G. Peter Matthews - Giuliano M. Laudone - Samuel Beckett • \\ Andrew Turner • Joachim Schoelkopf • Patrick A. C. Gane
}

Received: date / Accepted: date

\begin{abstract}
Microporous 'functionalised' calcium carbonate (FCC) has potential for use as a carrier for the controlled release of 'actives', by permeation and diffusion. We have investigated the nature of the FCC surface and the mechanism of adsorption of two typical actives, namely the anti-inflammatory drug aspirin and the flavour compound vanillin, from chloroform and aqueous ethanolic solutions. There is indirect evidence from the quantitative perturbation of Tóth isotherms that their adsorption is hindered by a stagnant diffusion layer of water trapped in the microporosity of the FCC. To complement previous studies of the surface of FCC, it was also tested with the cationic probe benzyltrimethylammonium bromide and the anionic probe sodium 2-naphthalenesulphonate. Experimental procedures were validated by comparison with adsorption onto ground calcium carbonate and high surface area talc.
\end{abstract}

Keywords Controlled drug delivery - Controlled flavour release $\cdot$ Functionalised calcium carbonate (FCC) · Vanillin . Aspirin

\section{Introduction}

\subsection{Functionalised calcium carbonate}

This work is a study of the adsorption properties of dissolved organic species onto 'functionalised' calcium carbonate (FCC). FCC comprises particles with intraparticulate porosity, which is microporous according to the pore size classification of Mays (2007). The adsorbates were the anti-inflammatory drug aspirin and the flavour compound vanillin, in chloroform and aqueous ethanolic solutions. The

\section{G. Peter Matthews}

Room 602, Davy Building, Drake Circus, Plymouth, Devon, PL4 8AA

Tel.: +44 1752584798

E-mail: p.matthews@plymouth.ac.uk
FCC surface was also tested with the cationic probe benzyltrimethylammonium bromide (BTMAB) and the anionic probe sodium 2-naphthalenesulphonate (Na2NS). Similarly sized particles of ground calcium carbonate (GCC) were used as a (null) control adsorbent. Finnish talc with a high surface area was used as a control to check the methodology of measuring the (null) adsorption of BTMAB onto FCC.

FCCs are produced by etching calcium carbonate particles and re-precipitating a modified surface structure with in-situ or externally supplied $\mathrm{CO}_{2}$ in the form of carbonic acid (Ridgway et al., 2004). To facilitate the in-situ production of $\mathrm{CO}_{2}$, acids such as phosphoric acid $\left(\mathrm{H}_{3} \mathrm{PO}_{4}\right)$ can be used. Variations in the etching process produce a range of morphologies with recrystallised surfaces, consisting of incorporated hydroxyapatite (HAP) in the case of phosphoric acid, which are dual porous with inter- and intraparticle porosity. FCCs have hydrophilic surfaces and exhibit both HAP and calcium carbonate crystalline structure (Gantenbein et al., 2012). FCC offers benefits over HAP because the current commercial methods used to produce HAP give a low yield mixed with several phases, so that the product is expensive and suitable only for small-scale and time-consuming production (Chen and Leng, 2015).

FCCs have a wide range of applications, particularly for the controlled release of actives such as drugs, plant protection chemicals, and food additives such as flavours. It has been proposed that the particles be utilised in various dosage forms, such as in chewing gum, a mouth or nasal spray, an inhaling device, a tablet, a lozenge, a trans-dermal patch and a powder (Pedersen and Andersen, 2012). FCCs can be tailored to suit a particular need; for example, plastically compressible FCC grades are available in the case where flavour release may require a compressive destruction of the inert carrier in the mouth combined with controlled release in order to excite the recipient's senses with flavour (Gane et al., 2006). FCCs are pharmaceutical grade analogues of 
"This is the author's accepted manuscript. The final published version of this work is published by Springer in Adsorption, 2017 available at: http://dx.doi.org/10.1007/s10450-017-9880-7 . This work is made available in accordance with the publisher's policies. Please refer to any applicable terms of use of the publisher.”

MCCs (modified calcium carbonates) designed for the paper industry, particularly for coatings designed for ink-jet printing (Ridgway et al., 2004). MCCs are normally treated with $<1 \%$ polyacrylate dispersant to facilitate transport and application (Alm et al., 2010; Gribble et al., 2010), whereas FCCs are free of organic chemicals.

1.2 Adsorption in the context of the controlled delivery of actives

The adsorption processes of drugs onto their delivery surfaces are important, since adsorption is the basic mechanism most commonly used for the loading of drugs onto surfaces such as nanodiamonds (Mochalin et al., 2013). Unwanted adsorption onto a surface may compromise the intended therapeutic benefit, driving up dosage levels, thus increasing treatment costs (Tzannis et al., 1997). A study by Mochalin et al. (2013) has also shown that the adsorption of poorly soluble drugs onto the surface of their nanodiamond particles may be suitable in overcoming the poor bioavailability of the drugs. They also state that the monolayer capacity for a particular drug on their surface must be determined in order to gain full advantage of the mechanism while avoiding excessive loading in order to minimise potential leakage of dangerous drugs. Pinholt et al. (2011) found that it is important to study the adsorption of peptide and protein drug products, as proteins are known to interact with interfaces of the particulate delivery system in which the effects are often irreversible adsorption and structural changes of the proteins. Kojima and Watanabe (2012) have studied HAP as a biomaterial and the adsorption and desorption of two bioactive proteins (cytochrom c and insulin) onto its surface; they concluded that HAP is a potential protein carrier with controlled release. A study by Neuvonen et al. (1984) has shown that the bioavailability of both aspirin and quinidine sulphate was significantly reduced when ingested with activated charcoal, and that a significant desorption of aspirin, but not that of quinidine, was subsequently detected. Tsuchiya and Levy's 1972 paper suggests that it may be possible to make reasonable predictions concerning the relative antidotal effectiveness of activated charcoal in humans on the basis of appropriate in vitro adsorption studies. Sellers et al. (1977) conducted comparative in vitro studies to determine the adsorption characteristics of 12 drugs onto activated charcoal in order to remediate any poorly absorbed drugs. They concluded that activated charcoal would not be helpful. Such diverse studies demonstrate that when a new drug delivery vehicle is being proposed, an investigation into the adsorptive properties of its surfaces is of paramount importance.

\subsection{Adsorbates}

Figure 1 shows the molecular structures of the four adsorbates used in this work. Benzyltrimethylammonium bromide (BTMAB) and sodium 2-naphthalene sulphonate (Na2NS) are charged, water soluble species, used as cationic and anionic probes, respectively.

Vanillin, a common and popular flavour compound readily soluble in ethanol, was chosen as the flavour species to adsorb onto the FCC in order to provide further insight into the mechanism of release in diffusion studies (Levy et al., 2015). It is solid at room temperature, thereby having potential to be loaded into the pores of an FCC in future work (Preisig et al., 2014). Although the use of aqueous solutions would be more applicable to biological situations, in this work adsorption from chloroform and ethanol/water solutions was studied, in order to facilitate loading of the vanillin onto the FCC, and to avoid the need for aqueous buffering which could interfere with the adsorption processes.

As an example drug, the adsorption of acetylsalicylic acid (aspirin) from aqueous ethanolic solutions is reported. Aspirin is a widely studied non-steroidal anti-inflammatory, antipyretic and analgesic drug (Vane and Botting, 2003).

\subsection{Surface properties of substrates}

FCC is an amphoteric material typically showing electrochemical charge values of +22 and $-17 \mu \mathrm{Eq} \mathrm{g}^{-1}$ in a polyelectrolyte titration versus the anionic polyelectrolye poly(vinyl sulfate) potassium salt (KPVS) and the cationic polyelectrolyte poly(diallyldimethylammonium chloride) (Gantenbein et al., 2012). Previous research and zeta potential measurements onto FCC surfaces have yielded complex results, suggesting that there must be both cationic and anionic surface adsorption sites (Gribble et al., 2010). The material is not suitable for measurements of its cation exchange capacity (CEC).

\subsection{Previous studies of adsorption onto minerals}

Gane et al. (2000) have shown that all dyes with a cationic charge show a strong adsorption onto anionically dispersed GCC. This indicates that coulombic forces contribute the strongest part of the adhesion force and is in agreement with the adsorbing mechanisms of ink jet dyes. Acidic dye molecules have a significantly weaker adhesion mechanism in which it is assumed that the $\mathrm{COOH}$-groups probably interact with free surface of the calcium carbonate. Dyes with an anionic colorant revealed no adsorption onto slightly anionically dispersed calcium carbonate surfaces, suggesting the possible existence of shielded cationic sites (Lamminmäki et al., 2011). In practice, ink jet dyes are usually anionic, and 
"This is the author's accepted manuscript. The final published version of this work is published by Springer in Adsorption, 2017 available at: http://dx.doi.org/10.1007/s10450-017-9880-7 . This work is made available in accordance with the publisher's policies. Please refer to any applicable terms of use of the publisher.”

a)<smiles>C[N+](C)(C)Cc1ccccc1</smiles>

b)<smiles>[O-]S([O-])([O-])c1ccc2ccccc2c1</smiles>

c)<smiles>CC(=O)Oc1ccccc1C(=O)O</smiles>

d)<smiles>COc1cc(C=O)ccc1O</smiles>

Fig. 1 Molecular structures of the four adsorbates: a) BTMAB, b) Na2NS, c) aspirin and d) vanillin.

ink jet paper coatings must therefore be cationised, at high cost.

Kallio et al. (2006) studied the adsorption of Food Black 2 (a diazo dye) onto coating pigments from polar liquids, such as water and ethanol, and from non-polar cyclohexane. They similarly concluded that electrostatic interactions are the predominating driving force for adsorption onto a variety of minerals, such as kaolin, calcium carbonate, and talc. These interactions are hydrophobic in the case of talc.

With respect to drugs, Mahato and Narang (2012) have found that adsorption generally increases as the ionization of the drug is suppressed, so that the extent of adsorption reaches a maximum when the drug is completely un-ionized. For amphoteric compounds, adsorption is at a maximum at the isoelectric point. $\mathrm{pH}$ and solubility effects act in concert since the un-ionized form of most drugs in aqueous solution has a low solubility. The physicochemical nature of the adsorbent affects the rate and extent of adsorption through changes in the molecular forces of attraction between the adsorbate and the adsorbent. Other work on the effect of changing the solvent showed that adsorption of actives onto calcium carbonate was irreversible from chloroform, but not from ethanol (Misra, 1991).

\subsection{FCC microporosity}

A recent work on diffusion from the microporosity of FCC involved intruding samples with mercury up to $400 \mathrm{MPa}$ applied pressure, and deconvoluting their percolation characteristics with PoreXpert ${ }^{\circledR}$ (Levy et al., 2015). The software generated a void network of the inter- and intraparticle pore space with the same mercury percolation characteristic as the FCC sample. Figure 2 shows a 'unit cell' of the intraparticle void space, with features ranging from 4.2 to $178 \mathrm{~nm}$ in diameter or edge size. The solid phase is shown transparent, and the voidage shown solid. Pores with textured surfaces are void clusters with sizes undifferentiated by the mercury porosimetry. There are periodic boundary conditions at each surface of the unit cell, equivalent to each unit cell connecting to infinite replicates in each Cartesian direction. The structuring has been highlighted by intruding 50 percent mercury by volume (shown grey) from the top surface in the direction shown. It can be seen that the percolation front is relatively uniform with respect to distance from the top surface. Correspondingly diffusion of the adsorbate into the sample prior to adsorption, and out from the body of the sample, would also depend primarily on depth from the surface rather than any small-scale lateral variations.

\subsection{Isotherms}

We use the standard definition of adsorption density $(\Gamma)$ (Pan and Liss, 1998):

$\Gamma=\frac{\left(C_{0}-C_{\mathrm{eq}}\right) V_{\text {total }}}{W_{\text {solid }}}$

where $C_{0}$ is the initial concentration of adsorbate in solution, $C_{\text {eq }}$ is the supernatant concentration after adsorption has taken place, $V_{\text {total }}$ is the volume of solution of concentration $C_{0}$ used in an adsorption experiment, and $W_{\text {solid }}$ is the weight of the adsorbent used during the adsorption experiment.

In this work, adsorption is described by the Tóth isotherm. This isotherm has the correct functionality at both limits as $C_{\mathrm{eq}} \rightarrow 0$ and $C_{\mathrm{eq}} \rightarrow \infty$, and has previously proved useful for fitting isotherms of porous adsorbents (Rudzinski and Everett, 1992). It has the form:

$\Gamma=\Gamma_{\max }\left(\frac{\left(K_{\mathrm{L}} C_{\mathrm{eq}}\right)^{m}}{1+\left(K_{\mathrm{L}} C_{\mathrm{eq}}\right)^{m}}\right)^{1 / m}$

where $\Gamma_{\max }$ is the adsorption at maximum equilibrium concentration solution $C_{\mathrm{eq}}$, and $K_{\mathrm{L}}$ is the Langmuir constant. The fitting parameter $m$, loosely associated with surface heterogeneity when $m \neq 1$, yields the Langmuir equation when $m=1$. In the present work, the Tóth isotherm is used to describe adsorption of aspirin and vanillin from ethanol onto FCC. In the presence of up to $20 \%$ by volume of added water it becomes:

$\Gamma=f^{\prime \prime}\left(\Gamma_{\max }\right)\left(\frac{\left[K_{\mathrm{L}} f^{\prime}\left(C_{\mathrm{eq}}\right)\right]^{m}}{1+\left[K_{\mathrm{L}} f^{\prime}\left(C_{\mathrm{eq}}\right)\right]^{m}}\right)^{1 / m}$

where the functions $f^{\prime}$ and $f^{\prime \prime}$ describe the effect of water on $C_{\mathrm{eq}}$ and $\Gamma_{\max }$ respectively. 
"This is the author's accepted manuscript. The final published version of this work is published by Springer in Adsorption, 2017 available at: http://dx.doi.org/10.1007/s10450-017-9880-7 . This work is made available in accordance with the publisher's policies. Please refer to any applicable terms of use of the publisher.”

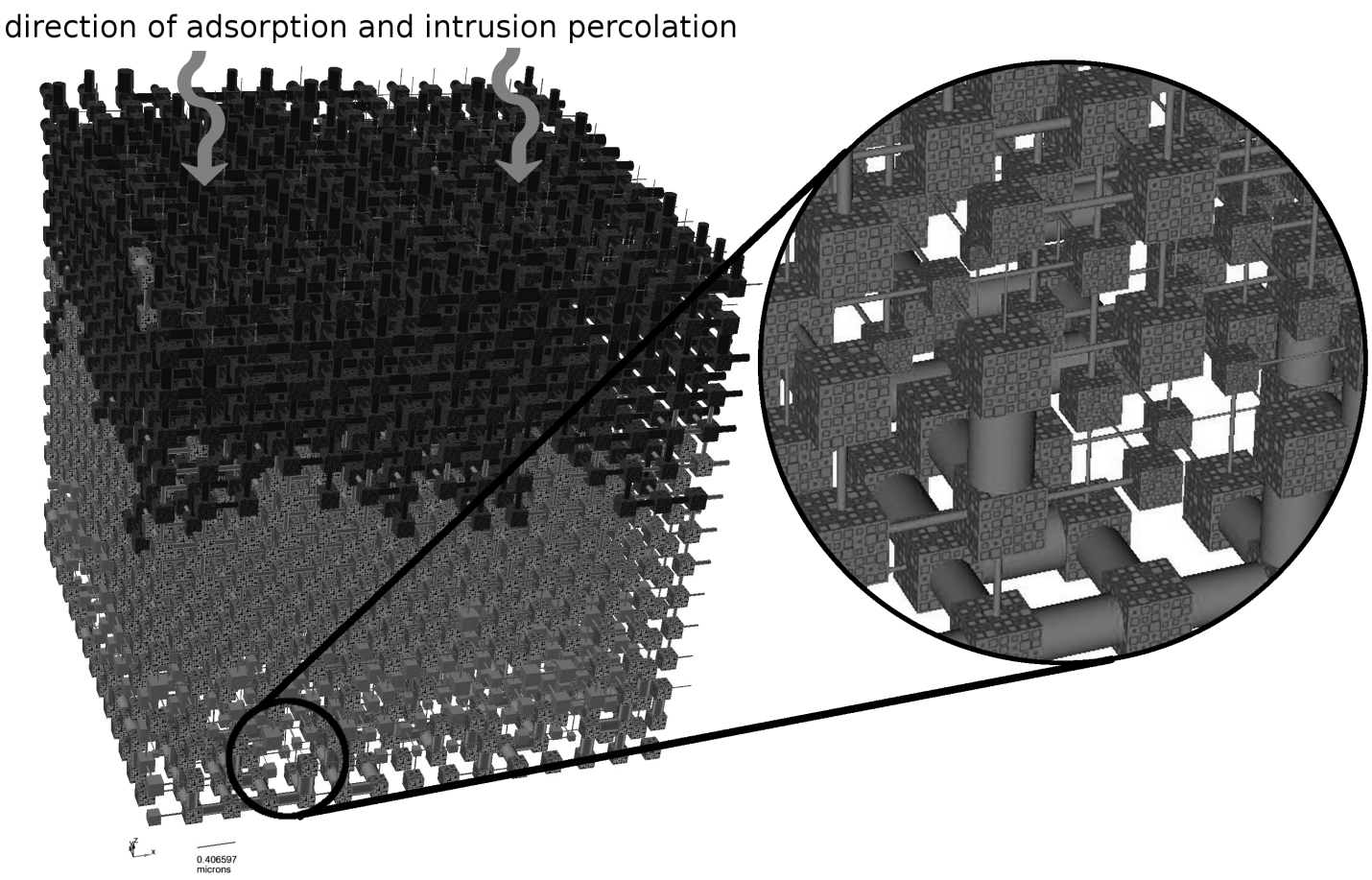

Fig. 2 PoreXpert ${ }^{\circledR}$ representation of the microporous intraparticle void space of FCC 03, as explained in the text. Scale bar bottom left: $407 \mathrm{~nm}$. A colour version of the Figure is provided in the Supplementary Material.

\section{Materials}

\subsection{Minerals}

The GCC and various grades of FCC samples were provided by Omya International AG (Oftringen, Switzerland). The GCC was Italian dry ground calcium carbonate prepared with a small amount of dry grinding aid, far too little to block the surface, but sufficient to influence moisture adsorption.

The properties of the minerals are listed in Table 1. Particle sizes were measured with a Malvern Master Sizer 2000, which measured the static light scattering of particles dispersed in water, interpreted using the Mie approximation (Mie, 1908). They are expressed in Table 1 as $d_{50}$ values, namely the median particle diameters at which $50 \%$ by volume of the sample is finer than the stated value. The $d_{50}$ value of $7.7 \mu \mathrm{m}$ for GCC is somewhat higher than its stated $d_{50}$ of $5 \mu \mathrm{m}$ based on Stokes' Law sedimentation in aqueous suspension. Surface areas were measured with a Micromeritics Gemini V surface area analyzer. The morphologies are explained in a previous publication (Levy et al., 2015), which also details the measurement of intra-particle porosities, and are shown as scanning electron micrographs in Figure 1 of the Supplementary Material.

\subsection{Adsorbates and solvents}

The vanillin (4-hydroxy-3-methoxybenzaldehyde; >99\%) and chloroform (HPLC grade stabilised with amylene) were supplied by Acros Organics. The absolute ethanol (HPLC grade) was from Fisher Scientific. BTMAB (>97\%) and aspirin (acetylsalicylic acid; >99\%) were supplied by Aldrich Chemistry (Sigma-Aldrich), Na2NS $(>99 \%)$ by FlukaAnalytical (Sigma-Aldrich), and the orthophosphoric acid (85\%) by CPR Rectapur.

\section{Methods}

\subsection{Vanillin adsorption from ethanol and chloroform}

$\lambda_{\max }$ for vanillin in ethanol was found to be $279 \mathrm{~nm}$, and $275 \mathrm{~nm}$ for vanillin in chloroform. The upper limit of quantification concentration was $40 \mathrm{mg} \mathrm{dm}^{-3}$. The vanillin stock solutions were prepared via serial dilution of a $1 \mathrm{~g} \mathrm{dm}^{-3}$ stock solution using borosilicate glass bulb pipettes.

An accurately known amount of approximately $0.2 \mathrm{~g}$ of FCC was weighed (Mettler Toledo XP504) into a $50 \mathrm{~cm}^{3}$ polypropylene plastic centrifuge tube, after which $25 \mathrm{~cm}^{3}$ of the desired concentration of vanillin was pipetted, using a borosilicate glass bulb pipette, into the centrifuge tube. The tube was then shaken briefly and vigorously by hand before being placed on a reciprocal shaker (Stuart SSL2) for 1 hour at 205 strokes per minute. It was then centrifuged (Centaur 2, 
"This is the author's accepted manuscript. The final published version of this work is published by Springer in Adsorption, 2017 available at: http://dx.doi.org/10.1007/s10450-017-9880-7 . This work is made available in accordance with the publisher's policies. Please refer to any applicable terms of use of the publisher.”

Table 1 Properties of the mineral particles. Particle sizes are expressed as $d_{50}$ values, namely the median particle diameters at which $50 \%$ by volume of the sample is finer than the stated value.

\begin{tabular}{|c|c|c|c|c|c|c|c|}
\hline Sample & $\begin{array}{c}\text { Particle } \\
\text { size } \\
d_{50} \\
/ \mu \mathrm{m}\end{array}$ & $\begin{array}{l}\text { Surface } \\
\text { area } \\
/ \mathrm{m}^{2} \mathrm{~g}^{-1}\end{array}$ & $\begin{array}{c}\begin{array}{c}\text { Overall } \\
\text { porosity }\end{array} \\
1 \%\end{array}$ & $\begin{array}{c}\text { Intra- } \\
\text { particle } \\
\text { porosity } \\
/ \%\end{array}$ & $\begin{array}{c}\text { Hydroxy- } \\
\text { apatite } \\
\text { content } \\
/ \%\end{array}$ & $\begin{array}{c}\text { Calcium } \\
\text { carbonate } \\
\text { content } \\
/ \%\end{array}$ & Morphology \\
\hline FCC TP & 7.0 & 55.4 & 61.6 & 25.9 & 51 & 49 & Roses \\
\hline FCC 02 & 4.9 & 54.1 & 61.2 & 24.4 & 43 & 57 & Roses \\
\hline FCC 03 & 3.1 & 33.5 & 49.3 & 10.4 & 14 & 86 & Caviar \\
\hline FCC 06 & 5.5 & 141.5 & 67.3 & 25.2 & 85 & 15 & Brain \\
\hline FCC 07 & 6.3 & 48.5 & 57.8 & 12.3 & 13 & 87 & Caviar \\
\hline FCC 12 & 10.0 & 81.0 & 70.1 & 29.6 & 50 & 50 & Flaky eggs \\
\hline FCC 13 & 23.5 & 66.0 & 53.4 & 37.0 & 50 & 50 & Golf balls \\
\hline GCC (control) & 7.7 & 1.53 & 22.2 & 0 & 0 & 97.5 & $\begin{array}{l}\text { Heterogeneous } \\
\text { blockey }\end{array}$ \\
\hline Finnish talc (control) & 5.02 & 45.3 & - & 0 & 0 & 0 & $\begin{array}{l}\text { High aspect ratio } \\
\text { plate-like }\end{array}$ \\
\hline
\end{tabular}

Sanyo, MSE) for 15 minutes at 3000 revolutions per minute $(1507 \mathrm{~g}$ ). The supernatant was then decanted into a clean and stoppered conical flask. Supernatants with initial concentrations $\left(C_{0}\right)$ above $40 \mathrm{mg} \mathrm{dm}^{-3}$ were diluted by a factor of $10 \mathrm{in} 100 \mathrm{~cm}^{3}$ volumetric flasks. The supernatants were then analysed by UV-vis spectroscopy (Hewlett Packard, now Agilent, 8452 ultraviolet-visible spectrometer).

In the case of adsorption experiments that involved a portion of the overall $25 \mathrm{~cm}^{3}$ volume of vanillin in ethanol being replaced by water, $20 \mathrm{~cm}^{3}$ of the desired concentration of vanillin was pipetted using a borosilicate glass bulb pipette, and the remaining $5 \mathrm{~cm}^{3}$ of water and vanillin in ethanol solution was pipetted in using a Thermo Scientific Finnpipette $(100-1000 \mu \mathrm{l})$. The volumetric proportions of water in ethanol ranged from $1 \%$ to $20 \%$.

For each concentration of adsorbate used in these adsorption experiments, another control experiment was carried out without any adsorbent sample but with the same concentration of adsorbate. The concentration of adsorbate in the supernatant with mineral sample ( $C_{\text {eq }}$ in Equation (1)) was subtracted from the concentration of adsorbate in the supernatant of the control sample $\left(C_{0}\right)$ in order to take into account any experimental artefacts, such as adsorption to the walls of the plastic centrifuge tube.

As an additional control experiment, adsorption onto FCC from ethanol was measured with constant $C_{0}$ (30 $\mathrm{mg} \mathrm{dm}^{-3}$ ) but varying $W_{\text {solid. }}$ The results of three experimental replicates were obtained, and showed no nonproportionate effect of substrate weight between 0.025 and $0.21 \mathrm{~g}$.

\subsection{BTMAB and Na2NS adsorption}

The wavelength of maximum spectral absorbance $\left(\lambda_{\max }\right)$ for BTMAB in water was found to be $262 \mathrm{~nm}$, and $274 \mathrm{~nm}$ for Na2NS. The upper limit for quantification of concentration by the spectrophotometer was $1 \mathrm{~g} \mathrm{dm}^{-3}$ and $0.1 \mathrm{~g} \mathrm{dm}^{-3}$ for BTMAB and Na2NS respectively. Electrolyte solutions, as specified below, were used to decrease the thickness of the electrical double layer and increase the probability of interactions between any surface charge on the mineral and the adsorbate.

An accurately known amount of approximately $2.0 \mathrm{~g}$ of FCC was weighed (Mettler Toledo Classic AB304-5 balance) into a $50 \mathrm{~cm}^{3}$ polypropylene plastic centrifuge tube, after which $25 \mathrm{~cm}^{3}$ of the desired concentration of adsorbate was pipetted, using a borosilicate glass bulb pipette, into the centrifuge tube. The tube was then shaken briefly and vigorously by hand. To ensure complete equilibration between adsorbate and adsorbent, the tube was then placed in a ceramic cylinder and rolled for 1 hour at around 100 revolutions per minute (Alpine Augsburg 1/25 LK). The tubes were then centrifuged (Rotina 420, Hettich Zentrifugen) for 6 minutes at 4500 revolutions per minute $(4166 g)$. The supernatant was then processed through a $0.2 \mu$ m regenerated-cellulose syringe filter (Sartorius RC25) into a clean polyethylene bottle. The $\mathrm{pH}$ of the supernatants was recorded (Mettler Toledo SevenMulti $\mathrm{pH}$ meter) before being diluted for analysis. In order to carry out the spectrophotometric analysis (Perkin Elmer Lambda 2 ultraviolet-visible spectrophotometer), an aliquot of supernatant was pipetted into a quartz cuvette along with an aliquot of the solvent (using Eppendorf and Rainin pipettes) to bring the absorbance of the supernatant within the calibration range.

\section{Results}

\subsection{Aspirin adsorption}

The adsorption of aspirin onto FCC from ethanolic solutions with various relative concentrations of water (relative permittivity $\left.\epsilon_{\mathrm{r}}=80.1\right)$ is shown in Figure 3. Three different 
"This is the author's accepted manuscript. The final published version of this work is published by Springer in Adsorption, 2017 available at: http://dx.doi.org/10.1007/s10450-017-9880-7 . This work is made available in accordance with the publisher's policies. Please refer to any applicable terms of use of the publisher.”

Table 2 Tóth isotherm parameters from Equations 2, 3, and 5.

\begin{tabular}{ccccc}
\hline $\begin{array}{c}\text { Adsorbate } \\
\text { Substrate }\end{array}$ & aspirin & vanillin & vanillin & BTMAB \\
Solvent & aqueous ethanol & aqueous ethanol & chloroform & water \\
\hline$\Gamma_{\max }\left(\mathrm{mg} \mathrm{g}^{-1}\right)$ & 16.0 & 5.0 & 15.3 & 25.8 \\
$K_{\mathrm{L}}\left(\mathrm{dm}^{3} \mathrm{mg}^{-1}\right)$ & 0.30 & 0.57 & 0.03 & 11.35 \\
$m$ & 1.00 & 0.58 & 0.50 & 0.26 \\
$A$ & 0.0035 & 0.0020 & - & - \\
$B$ & 0.7 & 1.7 & - & - \\
\hline
\end{tabular}

stock solutions were used, and up to seven replicates were measured for each $C_{\text {eq }}$, with error bars as shown, which correspond to one standard deviation $\left(\sigma_{\text {devn }}\right)$. Also shown are the corresponding modified Tóth isotherms (equation 3) which are discussed below. In this case, $m=1$, i.e. the adsorption of aspirin from pure ethanol follows a Langmuir isotherm.

\subsection{Vanillin adsorption}

The adsorption of vanillin onto FCC from aqueous ethanolic solutions is shown in Figure 4, for three replicates with error bars $\left(\sigma_{\text {devn }}\right)$ as shown. Also shown are the corresponding modified Tóth isotherms (Table 2).

The adsorption of vanillin from chloroform $\left(\epsilon_{\mathrm{r}}=4.8\right)$ was higher than from the more polar ethanol $\left(\epsilon_{\mathrm{r}}=24.3\right)$ (Figure 4). It was found that there was a minimal difference in the adsorption characteristics of vanillin from ethanol between different grades of FCC.

\subsection{Adsorption onto GCC}

GCC was used as a control substrate. No adsorption was observed of any of the species studied, from any of the solvents used.

\subsection{BTMAB and Na2NS adsorption}

There was a lack of adsorption of BTMAB and Na2NS onto FCC 13 in the presence of $0.1 \mathrm{M} \mathrm{NaCl}$. Furthermore, there was no adsorption of either probe in the presence of $2 \mathrm{M}$ $\mathrm{NaCl}$, or in $0.005 \mathrm{M} \mathrm{H}_{3} \mathrm{PO}_{4}$ electrolyte solutions, or in ultrapure water. The lack of adsorption at increased concentration of electrolytes showed that adsorption did not occur even when the electric double layer at the surface of the FCC was suppressed. The null results in ultrapure water showed that the electrolyte was not itself interfering with adsorption. The use of the $\mathrm{H}_{3} \mathrm{PO}_{4}$ solution provided a check of whether the FCC surface could be re-activated, again showing a null result.

The $\mathrm{pH}$ of the BTMAB and Na2NS stock solutions was in the range 5.7-6.2. The $\mathrm{pH}$ of the supernatants involving FCC as the adsorbent was in the range 7.9-8.3, and the $\mathrm{pH}$ of the supernatants involving talc as the adsorbent was in the range 8.6-8.9. The supernatant $\mathrm{pH}$ rose after an adsorption experiment because of the very slight solubility of the calcium carbonate.

It was necessary to confirm that the null results were not due to an error in experimental procedure. Adsorption of BTMAB onto Finnish talc with a high surface area of $45.3 \mathrm{~m}^{2} \mathrm{~g}^{-1}$ in $0.1 \mathrm{M} \mathrm{NaCl}$ solution did produce an isotherm. Error bars were calculated, based on an estimated accuracy of $1 \%$ in determining the BTMAB concentration. A Tóth adsorption isotherm passing through all such error bars had the parameters shown in Table 2. It was found that there was no adsorption of Na2NS onto talc. The maximum observed adsorption of $9.2 \mathrm{mg} \mathrm{g}^{-1}$, slowly asymptoting to a $\Gamma_{\max }$ of $25.8 \mathrm{mg} \mathrm{g}^{-1}$ (Table 2) is in accord with the maximum adsorbance of $2.14 \mathrm{mg} \mathrm{g}^{-1}$ onto talc with $33 \%$ chlorite observed by Charnay et al. (2001).

\section{Discussion}

5.1 Adsorption of aqueous and ethanolic aspirin and vanillin onto FCC

The hydrophobic moieties associated with aspirin and vanillin may well play a role in the way they adsorb. Misra (1988) stated that 'a coupling agent possessing a hydrogenbonding moiety could effectively interact with the adsorbed water on the apatite substrate. The agent will be easily displaced from the surface by water or any other hydrogenbonding solvent if it does not possess hydrophobic moieties concomitantly with its hydrogen-bonding groups.'

In ethanolic solutions, it is possible that the -OH functional group on the ethanol orders itself around the HAP surface, leaving the $-\mathrm{CH}_{3}$ pointing away from the surface, 
“This is the author’s accepted manuscript. The final published version of this work is published by Springer in Adsorption, 2017 available at: http://dx.doi.org/10.1007/s10450-017-9880-7 . This work is made available in accordance with the publisher's policies. Please refer to any applicable terms of use of the publisher.”

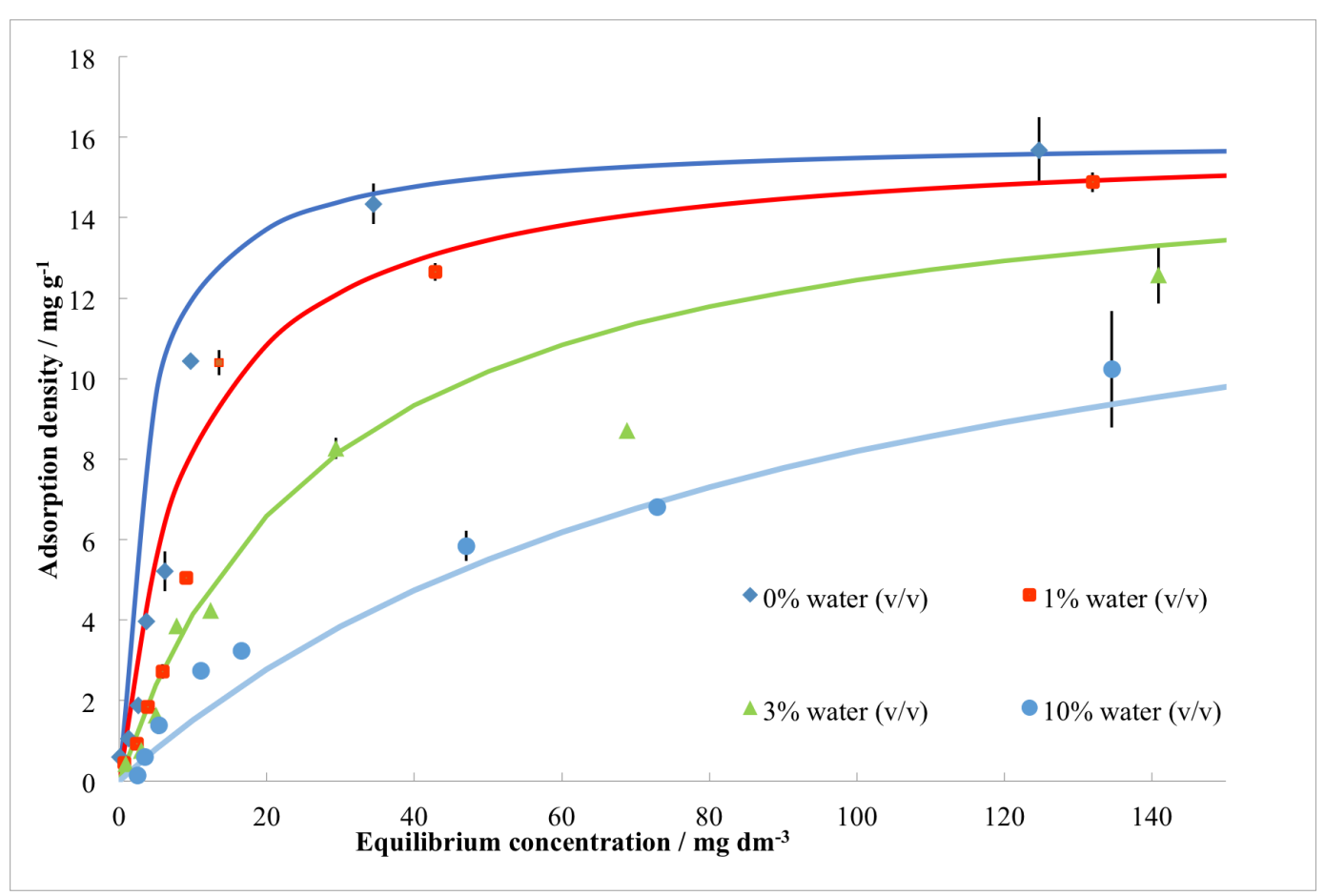

Fig. 3 Aspirin adsorption from aqueous ethanol solutions onto FCC with relative volumetric percentage of water as shown and corresponding Tóth isotherms.

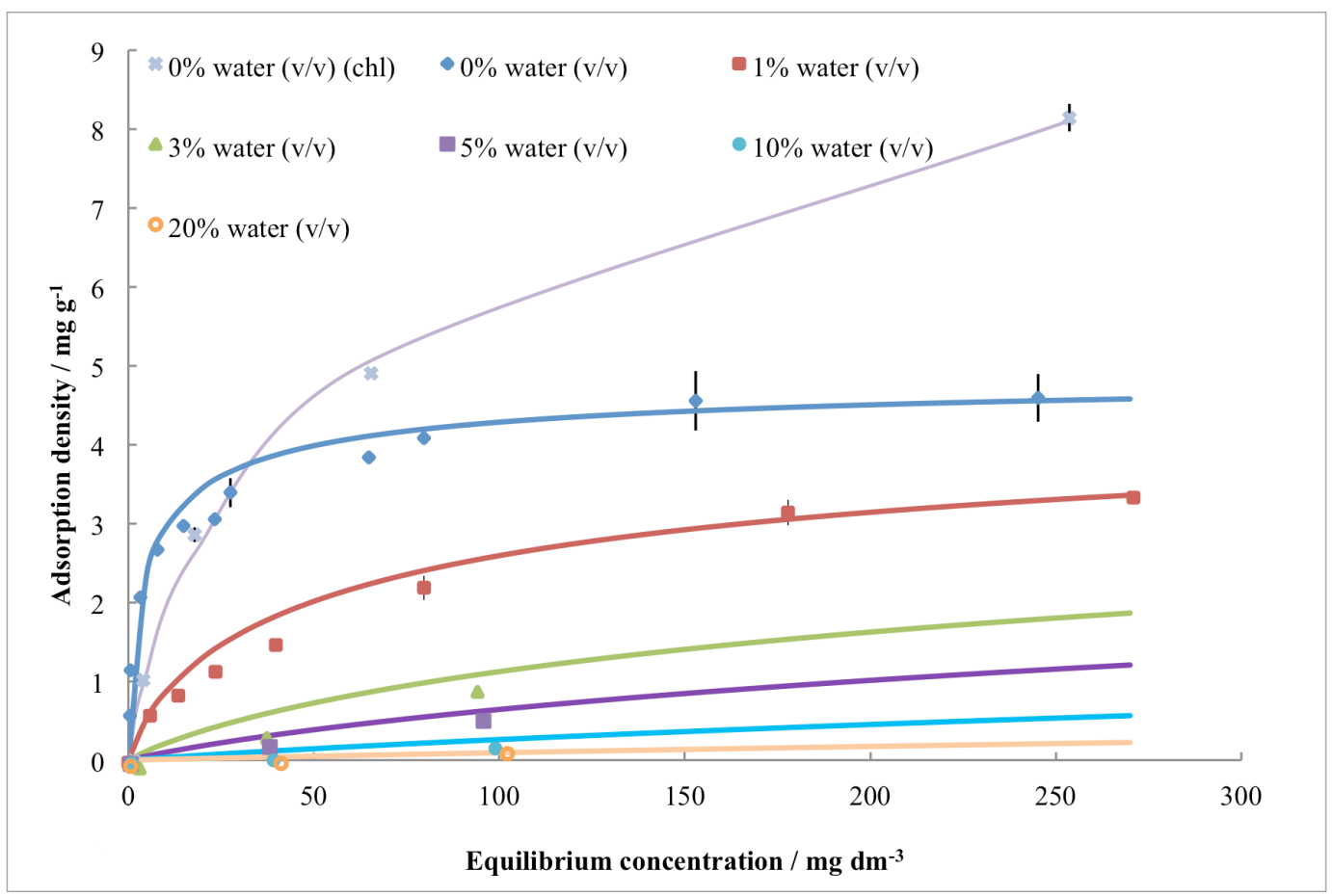

Fig. 4 Vanillin adsorption from aqueous ethanol solutions onto FCC with relative volumetric percentage of water as shown and corresponding Tóth isotherms; adsorption from chloroform is denoted with 'chl.' 
"This is the author's accepted manuscript. The final published version of this work is published by Springer in Adsorption, 2017 available at: http://dx.doi.org/10.1007/s10450-017-9880-7 . This work is made available in accordance with the publisher's policies. Please refer to any applicable terms of use of the publisher.”

thus creating effectively hydrophobic sites. As the aspirin or vanillin approaches the FCC surface it can orientate itself so that hydrophobic interactions take place. Such speculation is supported by the observation that the use of relatively apolar chloroform $\left(\epsilon_{\mathrm{r}}=4.8\right)$ as a solvent increases the amount of vanillin adsorbed (Figure 4 and Table 2).

Greater insights into the adsorption process can be made by estimating the surface coverage using the relation:

$S_{\mathrm{ads}}=\Gamma_{\max }^{\prime} \mathcal{N} \sigma$

Here $\Gamma_{\max }^{\prime}$ is the maximum adsorption (Table 2) expressed in moles per gram of substrate, $\mathcal{N}$ is the Avogadro number and $\sigma$ is the effective cross-sectional area of the adsorbing cation on the surface of the substrate. $\Gamma_{\max }^{\prime}$ is the asymptote of an imprecise extrapolation of the observed experimental data to infinite $C_{\text {eq }}$, and $\sigma$ is also an estimate, so the equation can only give a very approximate estimate of surface coverage $S_{\text {ads. }}$.

To calculate the surface coverage by vanillin, the volume of a known quantity of vanillin was measured by helium pycnometry (Thermo Scientific Pycnomatic ATC). From this, the volume of one vanillin molecule was deduced, and the corresponding cross-sectional area calculated as $0.44 \mathrm{~nm}^{2}$. It follows that the calculated surface covered of FCC TP by vanillin adsorption from ethanol was $9.53 \mathrm{~m}^{2} \mathrm{~g}^{-1}$, which is around $17 \%$ of the overall FCC TP surface area (Table 1). For adsorption from chloroform the surface coverage was calculated to be $26.33 \mathrm{~m}^{2} \mathrm{~g}^{-1}$, which is $48 \%$ of the FCC's surface area.

For aspirin, the spherical-equivalent volume of a molecule was calculated from the density of aspirin of 1.404 $\mathrm{g} \mathrm{cm}^{-3}$ as measured by Sun (2004) using helium pycnometry. The resulting cross-sectional area is $0.43 \mathrm{~nm}^{2}$. It follows that the calculated surface covered of FCC TP by aspirin adsorption from ethanol was $21.64 \mathrm{~m}^{2} \mathrm{~g}^{-1}$, which is $39 \%$ of the overall FCC TP surface area.

On the basis of work by Schatzberg (1967), the sphericalequivalent cross-sectional area of a water molecule is 0.49 $\mathrm{nm}^{2}$. It follows that in a solution of $1 \%$ water by volume, the water molecules in the current adsorption experiment would cover $410 \mathrm{~m}^{2}$, relative to an available surface area of only $11 \mathrm{~m}^{2}$ on the FCC. So there is more than enough water in the experiments to cover the entire surface of the FCC, and therefore water molecules do not simply block the adsorption sites to adsorption of vanillin.

When quantitatively explaining the effect of water on the Tóth adsorption isotherms, let us assume that the adsorption process must be analogous for both aspirin and vanillin. In the presence of water, the second derivative of the adsorption with respect to $C_{\mathrm{eq}}$ (i.e. curvature of the isotherm) reduces, and in the case of vanillin becomes negative as the relative water content is increased (Figures 3 and 4). As shown in these figures, it is found that to a realistic level of approximation,

$f^{\prime}\left(C_{\mathrm{eq}}\right)=C_{\mathrm{eq}}\left(\frac{A}{\left[\mathrm{H}_{2} \mathrm{O}\right]}\right)^{B}$

with the parameters shown in Table 2. Furthermore, it is found that there is no effect of water on $\Gamma_{\max }$, i.e. $f^{\prime \prime}=1$.

Therefore it is confirmed that the added water is not reducing the number of adsorption sites, in agreement with the calculation above. Instead, the results can be satisfactorily explained by assuming that there is a reduction in the effective flux of adsorbate reaching the FCC surface, caused by a stagnant layer of water trapped within the FCC microporosity, Figure 5. The orientation of the ethanol, described above, creates sites or zones which are effectively hydrophobic, shown as $x$ in the figure.

Crank (1975) in his Figure 4.2 shows that as time increases, then for the flux $Q_{t}$ at time $t$ through a stagnant diffusion layer of thickness $l, Q_{t} / l C_{1}$ becomes linearly proportional to $D t / l^{2}$, and therefore that at constant diffusion coefficient $D$ and bulk solution concentration $C_{1}, Q_{t} \propto l^{-1}$ as $t \rightarrow \infty$. If the thickness of this diffusion layer was directly proportional to the water concentration, then $Q_{t} \propto\left[\mathrm{H}_{2} \mathrm{O}\right]^{-1}$. In practice, it is found that $Q_{t}$ reduces approximately as $\left[\mathrm{H}_{2} \mathrm{O}\right]^{-0.7}$ for aspirin and $\left[\mathrm{H}_{2} \mathrm{O}\right]^{-1.7}$ for vanillin (Table 2). Inspection of Figure 3 shows that at higher $C_{\text {eq }}$, the adsorption density $\Gamma$ does not follow the perturbed Tóth isotherm exactly (equation 3 ). These discrepancies, and the fact that the exponents are not unity, are to be expected because the aqueous diffusion layer exists within a random tortuous mesoporosity, Figure 2 (Levy et al., 2015), rather than being in the form of a stable planar layer.

Mercury porosimetry of FCC detects voids as small as 4.2 $\mathrm{nm}$, subsection 1.6. The adsorption behaviour in such voids would probably differ from that shown in Figure 5, since the solvents within the voids may be partially orientationally ordered with lowered configurational entropy, and the voids would easily block. However, the PoreXpert unit cell derived from the porosimetry, Figure 2, contains very few of the smallest voids, and their contribution by volume is negligible.

\subsection{Lack of adsorption of aqueous BTMAB and Na2NS onto FCC}

In this work, no adsorption of BTMAB or Na2NS was observed from aqueous solution onto any grade of FCC. Lamminmäki et al. (2011) postulate that water adsorbs strongly to the surface of FCC via hydrogen bonding. Such a postulate is supported by the fact that the FCC is highly hydrophilic. The contact angle reported for $98 \%$ pure calcium carbonate is $10^{\circ}$ (Ren et al., 2003), and for hydroxyapatite is $31^{\circ}$ 
“This is the author’s accepted manuscript. The final published version of this work is published by Springer in Adsorption, 2017 available at: http://dx.doi.org/10.1007/s10450-017-9880-7 . This work is made available in accordance with the publisher's policies. Please refer to any applicable terms of use of the publisher.”

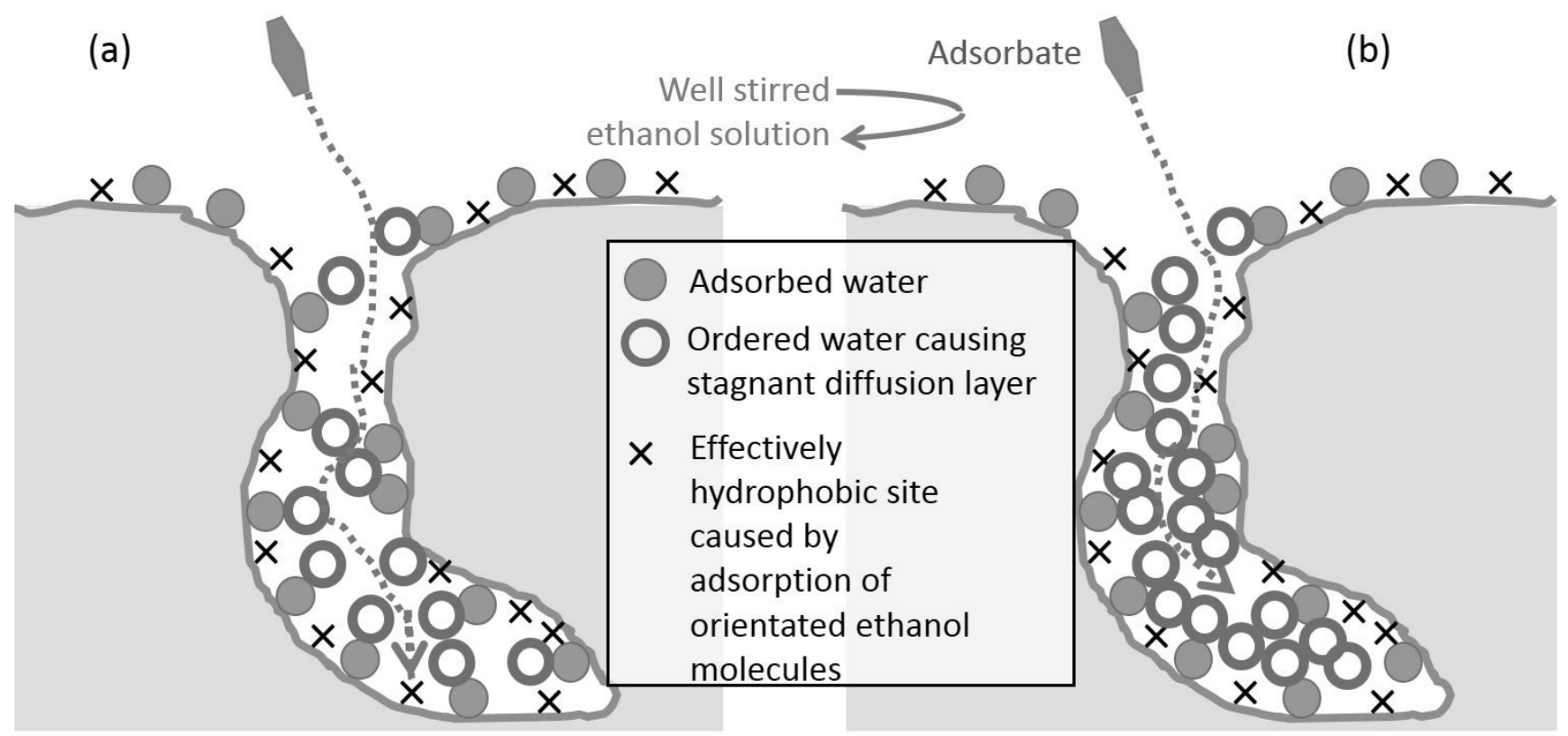

Fig. 5 Schematic diagram of the postulated mechanism for the adsorption of aspirin and vanillin onto FCC in the presence of (a) lower and (b) higher relative concentrations of water. The scale of the features is shown in Figure 2.

(Toriyama et al., 1995). As FCC is a composite of both of these materials, the FCC will also be hydrophilic. Direct observation of the contact angle of water onto FCC is not possible because water adsorbs onto the microporous surface so quickly (Tåg et al., 2010). FCC is likely to have at least a monolayer of physically adsorbed water when exposed in ambient air (Misra, 1988). However, any explanation based on surface properties does not encompass the observation that polyelectrolytes such as polyacrylate and poly(diallyldimethylammonium chloride) (PolyDADMAC) do adsorb onto FCC. An explanation could be that hydrophobic moieties are either forming hydrophobically linked micelles that are too large and wrongly oriented to adsorb, or, less likely, that tiny amounts of BTMAB and Na2NS are adsorbing and rendering the external surface hydrophobic and non-adsorbing.

\section{Conclusions}

We have demonstrated the adsorption properties of functionalised calcium carbonate (FCC) for aspirin and vanillin. FCC does not adsorb these species from aqueous solution. Unlike talc, which has a surface which can be characterised by the adsorption of BTMAB, the surface of FCC is amphoteric and characterisation of adsorption sites is much more elusive. However, we have demonstrated that by studying the adsorption of vanillin and aspirin from aqueous ethanolic solutions, a mechanism for the adsorption can be deduced which is quantitatively supported both by calculations of sur- face coverage and by the effect of water on the shapes of the adsorption isotherms.

The understanding of the mechanism of adsorption opens the way for innovative, tailored loading of actives onto FCC. It suggests that during controlled release, adsorption effects need not be taken into account. Additionally it is shown that there will be no interference with functional groups of the active, and the flavour or drug will be delivered without alteration of its efficacy or activity.

Acknowledgements The financial support of Omya International AG is gratefully acknowledged. We are grateful to Daniel Gerard and Cathy Ridgway of Omya International AG for measuring the surface areas of the substrates, and to their colleague Daniel Gantenbein for his advice. We recognise the early adsorption work onto FCC carried out by Samuel Wieck.

\section{References}

H.K. Alm, G. Ström, K. Karltröm, J. Schoelkopf, P.A.C. Gane, Effect of excess dispersant on surface properties and liquid interactions on calcium carbonate containing coatings. Nord. Pulp. Pap. Res. J. 25(1), 082-092 (2010)

C. Charnay, S. Lagerge, S. Partyka, Assessment of the surface heterogeneity of talc materials. J. Colloid Interf. Sci. 233(2), 250-258 (2001)

H. Chen, S. Leng, Rapid synthesis of hollow nano-structured hydroxyapatite microspheres via microwave transformation method using hollow $\mathrm{CaCO}_{3}$ precursor microspheres. Ceram. Int. 41(2, Part A), 2209-2213 (2015) 
“This is the author’s accepted manuscript. The final published version of this work is published by Springer in Adsorption, 2017 available at: http://dx.doi.org/10.1007/s10450-017-9880-7 . This work is made available in accordance with the publisher's policies. Please refer to any applicable terms of use of the publisher.”

J. Crank, The Mathematics of Diffusion, $2^{\text {nd }}$ edn., (Oxford University (Clarendon) Press), 1975

P.A.C. Gane, C.J. Ridgway, E. Barceló, Analysis of pore structure enables improved tablet delivery systems. Powder Technol. 169(2), 77-83 (2006)

P.A.C. Gane, G.P. Matthews, J. Schoelkopf, C.J. Ridgway, D.C. Spielmann, Fluid transport into porous coating structures: some novel findings. Tappi J. 83(5), 1-22 (2000)

D. Gantenbein, J. Schoelkopf, G.P. Matthews, P.A.C. Gane, The use of porous high surface area calcium carbonate for the adsorption of dissolved and colloidal substances from thermo mechanical pulp filtrates. Nord. Pulp. Pap. Res. J. 27(3), 631-638 (2012)

C.M. Gribble, G.P. Matthews, D. Gantenbein, A. Turner, J. Schoelkopf, P.A. Gane, Adsorption of surfactant-rich stickies onto mineral surfaces. J. Colloid Interf. Sci. 352(2), 483-90 (2010)

T. Kallio, J. Kekkonen, P. Stenius, Acid/base properties and adsorption of an azo dye on coating pigments. J. Dispersion Sci. Technol. 27(6), 825-834 (2006)

C. Kojima, K. Watanabe, Adsorption and desorption of bioactive proteins on hydroxyapatite for protein delivery systems. J. Drug Deliv. 2012(Article ID 932461), 4 (2012)

T.T. Lamminmäki, J.P. Kettle, P.A.C. Gane, Absorption and adsorption of dye-based inkjet inks by coating layer components and the implications for print quality. Colloid. Surface. A 380(1-3), 79-88 (2011)

C.L. Levy, G.P. Matthews, G.M. Laudone, C.M. Gribble, A. Turner, C.J. Ridgway, D.E. Gerard, J. Schoelkopf, P.A.C. Gane, Diffusion and tortuosity in porous functionalized calcium carbonate. Ind. Eng. Chem. Res. 54(41), 99389947 (2015)

R.I. Mahato, A.S. Narang, Pharmaceutical Dosage Forms and Drug Delivery, $2^{\text {nd }}$ edn. Pharmacy Education Series, (CRC Press, Taylor \& Francis Group), 2012

T.J. Mays, A new classification of pore sizes, ed. by F.R.R.J.R. P.L. Llewellyn, N. Seaton, vol. 160 (Elsevier, 2007), pp. 57-62

G. Mie, Beiträge zur optik trüber medien, speziell kolloidaler metallösungen. Ann. Phys-Berlin 330(3), 377-445 (1908)

D.N. Misra, Adsorption on hydroxyapatite: role of hydrogen bonding and interphase coupling. Langmuir 4(4), 953-958 (1988)

D.N. Misra, Adsorption and orientation of tetracycline on hydroxyapatite. Calcified Tissue Int. 48(5), 362-367 (1991)

V.N. Mochalin, A. Pentecost, X.-M. Li, I. Neitzel, M. Nelson, C. Wei, T. He, F. Guo, Gogotsi, Yury, Adsorption of drugs on nanodiamond: Toward development of a drug delivery platform. Mol. Pharmaceutics 10(10), 3728-3735 (2013)

P.J. Neuvonen, K.T. Olkkola, T. Alanen, Effect of ethanol and $\mathrm{pH}$ on the adsorption of drugs to activated charcoal: studies in vitro and in man. Acta Pharmacol. Toxicol. (Copenh) 236(1-3), 91-102 (1984)
G. Pan, P.S. Liss, Metastable-equilibrium adsorption theory: I. Theoretical. J. Colloid Interf. Sci. 201(1), 71-76 (1998)

K.M. Pedersen, J.B. Andersen, Particulate material for controlled release of active ingredients, Omya Development AG, 2012. Patent US 20120321751 A1

C. Pinholt, R.A. Hartvig, N.J. Medlicott, L. Jorgensen, The importance of interfaces in protein drug delivery - why is protein adsorption of interest in pharmaceutical formulations? Expert Opin Drug Deliv. 8(7), 949-964 (2011)

D. Preisig, D. Haid, F.J.O. Varum, R. Bravo, R. Alles, J. Huwyler, M. Puchkov, Drug loading into porous calcium carbonate microparticles by solvent evaporation. Eur. J. Pharm. Biopharm. 87(3), 548-558 (2014)

J. Ren, W. Wang, S. Lu, J. Shen, F. Tang, Characteristics of dispersion behavior of fine particles in different liquid media. Powder Technol. 137(1-2), 91-94 (2003)

C.J. Ridgway, P.A.C. Gane, J. Schoelkopf, Modified calcium carbonate coatings with rapid absorption and extensive liquid uptake capacity. Colloid. Surface. A. 54(1), 1-7 (2004)

W. Rudzinski, D.H. Everett, Adsorption of Gases on Heterogeneous Surfaces, $1^{\text {st }}$ edn., (Elsevier Ltd.), 1992

P. Schatzberg, Molecular diameter of water from solubility and diffusion measurements. J. Phys. Chem. A 71(13), 4569-4570 (1967)

E.M. Sellers, V. Khouw, L. Dolman, Comparative drug adsorption by activated charcoal. J. Pharm. Sci. 66(11), 1640-1641 (1977)

C. Sun, A novel method for deriving true density of pharmaceutical solids including hydrates and water-containing powders. J. Pharm. Sci. 93(3), 646-653 (2004)

C.-M. Tåg, M. Juuti, K. Koivunen, P.A.C. Gane, Dynamic water transport in a pigmented porous coating medium: Novel study of droplet absorption and evaporation by nearinfrared spectroscopy. Ind. Eng. Chem. Res. 49(9), 41814189 (2010)

M. Toriyama, Y. Kawamoto, T. Suzuki, Y. Yokogawa, K. Nishizawa, F. Nagata, Wettability of calcium phosphate ceramics by water. J. Ceram. Soc. Jpn. 103(1193), 46-49 (1995)

T. Tsuchiya, G. Levy, Relationship between effect of activated charcoal on drug absorption in man and its drug adsorption characteristics in vitro. J. Pharm. Sci. 61(14), 586-589 (1972)

S.T. Tzannis, W.J.M. Hrushesky, P.A. Wood, T.M. Przybycien, Adsorption of a formulated protein on a drug delivery device surface. J. Colloid Interface Sci. 189(2), 216-228 (1997)

J.R. Vane, R.M. Botting, The mechanism of action of aspirin. Thromb. Res. 110(5-6), 255-258 (2003) 